\title{
Implementing sustainable IT strategy: the case of Intel
}

\author{
Edward Curry ${ }^{1}$, Brian Donnellan ${ }^{2}$ \\ ${ }^{1}$ Digital Enterprise Research Institute, National University of Ireland, Galway, Ireland; \\ ${ }^{2}$ Innovation Value Institute, National University of Ireland, Maynooth, Ireland
}

Correspondence:

E Curry, Digital Enterprise Research Institute, National University of Ireland, IDA Business Park, Lower Dangan, Galway, Ireland.

Tel: +35391 492973;

Fax: +35391 495541;

E-mail: edcurry@acm.org

\begin{abstract}
Sustainable IT (Information Technology) involves the responsible management of resources (both IT and non-IT) encompassing environmental, economic, and social dimensions. Sustainable IT has the potential to be a significant contributor to an organization's sustainability strategy. In this teaching case, we examine what Intel IT has done to transform its operations with Sustainable IT, resulting in the avoidance of significant $\mathrm{CO}_{2}$ emissions and cost savings. This teaching case challenges the reader to analyze the Sustainable IT capability at Intel. The case includes insights into strategic and operational challenges of planning and managing Sustainable IT.
\end{abstract}

Journal of Information Technology Teaching Cases (2014) 4, 41-48. doi:10.1057/jittc.2014.2; published online 4 March 2014

Keywords: teaching case; sustainable IT; green IT; IT management

\section{B}

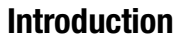
ill Guyon leads the Intel IT (Information Technology) Sustainability Program Office, with responsibility for coordinating sustainability initiatives and driving Sustainable IT within Intel. In the summer of 2012, Bill is looking to assess the progress achieved in the 4 years since the IT Sustainability Program Office was founded, and to develop a new IT Sustainability strategy for the next 5 years. The existing strategy has positioned Intel as a leader in the emerging area of Sustainable IT, achieved cost savings of over $\$ 114$ million, and avoidance of more than 87,500 metric tons of $\mathrm{CO}_{2}$ emissions. In recognition of these achievements, Intel IT has won a number of industrial awards for IT Sustainability.

Bill now needs to decide what the next strategic steps are for Sustainable IT at Intel. Can Intel further exploit the transformational and innovation potential of Sustainable IT within the organization and across the wider extended-enterprise? What opportunities do the next generation of Sustainable IT technologies present? Can Sustainable IT be a competitive advantage for Intel?

Bill must present the strategy to senior management (including the $\mathrm{CEO}, \mathrm{CIO}, \mathrm{COO}, \mathrm{CSO}$, and senior leaders from across the company) to secure commitment for the new IT Sustainability strategy. The strategy must detail why Intel should further develop the IT Sustainability capability and how it will deliver IT-enabled Sustainability benefits for both Intel IT and wider Intel Organization. The strategy needs a solid business case for sustainability covering the triple bottom line (Environmental, Social, and Economic), and will need to justify the required investment need for a long-term commitment to Sustainable IT. ${ }^{1}$

\section{Sustainability}

Sustainability now holds a significant position on the strategic agenda of many large corporations and government agencies. Sustainability is not only about Corporate Social Responsibility, Sustainability is an important business issue affecting new products and services, compliance, cost reduction opportunities, the organization's reputation, and revenue generation. Porter recognizes the role sustainability can play as part of an organization's Competitive Strategy with the concept of 'innovation offsets' - where companies can 'not only lower the net costs of meeting environmental regulations, but can lead to absolute advantages' over competitors (Porter and Linde, 1995). Banking on growing consumer demand for green products and services, some retailers have developed 'subbrands' with a green angle. Increasingly, manufacturers across industries will use green products and green corporate behavior as a way to appeal to this growing segment of customers. As organizations embrace sustainability agendas they will need to develop relevant capabilities to deliver on the promise. Many organizations think it requires a significant 
transformational change program, yet the ultimate goal is to embed sustainability into business-as-usual activities. IT departments that want to be key players within their organization's sustainability strategy will need to develop significant Sustainable IT capability.

\section{Sustainable IT}

Sustainable IT is the design, production, operation, and disposal of IT and IT-enabled products and services in a manner that is not harmful and may be positively beneficial to the environment during the course of its whole-of-life (Elliot, 2007). Sustainable IT requires the responsible management of resources (both IT and non-IT) encompassing environmental, economic, and social dimensions. The first wave of Sustainable IT, Greening of IT, aims to reduce the $2 \%$ of global Greenhouse Gas (GHG) emissions for which IT is responsible (Webb, 2008), by reducing the footprint of IT through actions such as improving the energy efficiency of hardware (processors and disk drives) and reducing waste from obsolete hardware. The second wave of Sustainable IT, Greening by IT, also called Green IT 2.0 (Murugesan and Laplante, 2011), is shifting the focus toward reducing the remaining $98 \%$ by focusing on the innovative use of IT in business processes to deliver positive sustainability benefits beyond the direct footprint of IT, such as monitoring a firm's emissions and waste to manage them more efficiently.

The potential of Greening by IT to reduce GHG emissions has been estimated at approximately $7.8 \mathrm{Gt} \mathrm{CO}_{2}$ of savings in 2020 , representing a $15 \%$ emission cut in 2020 and 600 billion ( $\$ 946.5$ billion) of cost savings (Webb, 2008). The use of IT for Greening will play a key role in the delivery of benefits that can alleviate at least five times the GHG footprint of IT itself (Enkvist and Rosander, 2007). To leverage these benefits, many corporate IT departments are now looking at developing Sustainable IT capabilities (Donnellan et al., 2011). Unfortunately, many organizations often do not exploit IT's full potential in their efforts to achieve sustainability. In this teaching case, we examine how Intel has transformed its operations with Sustainable IT, resulting in the avoidance of significant $\mathrm{CO}_{2}$ emissions and cost savings. The case includes insights into strategic and operational challenges of planning and managing a Sustainable IT program.

\section{Sustainability at Intel}

With emissions of 3,850,000 metric tons of $\mathrm{CO}_{2}$ in 2007, Intel Corporation knew it faced a long-term challenge to reduce its absolute environmental footprint. Left untouched, overall emissions would increase due to the growth of the company and the increasing complexity of its design and manufacturing processes. Recognizing that addressing these challenges would be a strategic priority for the company in the coming years, Intel chose to develop significant capabilities and a reputation for leadership in the area of sustainability. At the start of 2008, Intel CEO Paul Otellini set out an ambitious 5-year goal to reduce environmental impacts in key areas including energy efficiency, water conservation, and a $20 \%$ emissions reduction on 2007 levels by 2012 .

Intel CIO Diane Bryant saw an opportunity for Intel IT to play a key role in enabling Intel to achieve their corporate sustainability goals. She committed Intel IT to helping the business deliver their objectives through the use of
Sustainable IT to reduce the environmental impacts of IT operations, and help transform the overall Intel organization. Intel IT wanted to utilize Sustainable IT to:

- align all IT processes and practices with the core principles of sustainability, which are to reduce, reuse, and recycle; and

- find innovative ways to use IT in business processes to deliver sustainability benefits across the enterprise and beyond.

If Intel IT were going to deliver on this promise, the organization would need to develop significant Sustainable IT capability.

\section{Company background}

Intel Corporation is the largest semiconductor manufacturer in the world, with major facilities in the United States, Europe, and Asia. Intel has changed the world dramatically since it was founded on July 18, 1968; the company invented the microprocessor that made possible the first handheld calculators and personal computers (PCs). Founded by semiconductor pioneers Robert Noyce and Gordon Moore, Intel combines advanced chip design capability with a leading-edge manufacturing capability. Intel is a portmanteau of Integrated Electronics (though a common misconception is that 'Intel' is from the word intelligence).

By the early 21st century, Intel's microprocessors were found in more than $80 \%$ of PCs worldwide. The company's product line also includes motherboard chipsets, network interface controllers and integrated circuits, flash memory, graphic chips, embedded processors, and other devices related to communications and computing.

Sustainability is an important part of Intel's strategy and long-term goals. In economic terms, it brings value by helping to mitigate risk, save costs, protects brand value, and develops new products and market opportunities.

In environmental terms, Intel has a track record from the mid-1990s of taking a proactive approach to climate issues as illustrated in Figure 1. Intel also has a history of social responsibility toward its employees and the wider community with initiatives for safety, ethical work environments, worklife balance, supply-chain audits, and community service volunteer programs such as teacher training.

\section{The sustainability of Intel IT}

In 2008, Bryant established the IT Sustainability Program Office to better manage sustainability initiatives and to catalyze change within IT and across Intel and the industry (Curry et al., 2012). At the time, Intel IT provided compute and communication services to Intel's 83,500 employees across 150 sites in 61 countries around the world. Intel IT operations managed more than 200,000 devices (90k+ PCs, 20k+ Handhelds) and 103 Data Centers ( 100,000 servers). The key Sustainable IT challenge for Intel IT was how to manage their IT footprint and enable corporate sustainability practices. The first task of the office was to develop a sustainability strategy to educate and provide leadership to the organization on the principles and importance of sustainable business practices. 


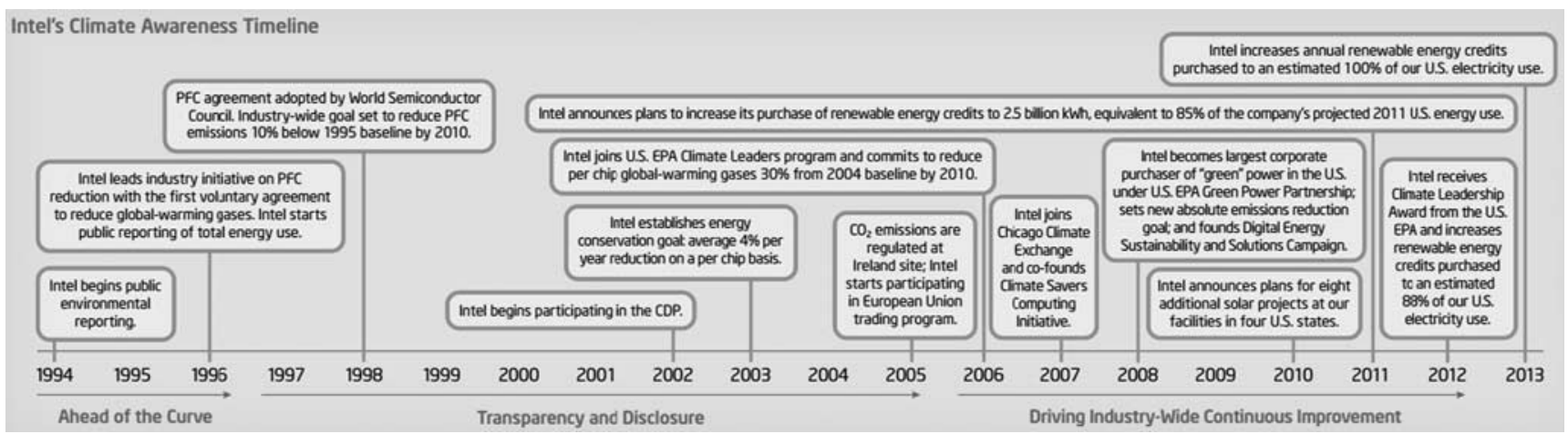

Figure 1 Since the mid-1990s, Intel has taken voluntary steps and set aggressive goals to reduce their greenhouse gas emissions. (Source: Intel ARP, 2012).

\section{Aligning IT and corporate goals}

When Intel IT wanted to define their Sustainable IT strategy, their first action was to obtain management commitment toward their sustainability goals. The Intel organization was already proactively addressing sustainability with an established corporate goal of reducing their carbon footprint by $20 \%$ by 2012 from 2007 levels. Intel IT worked with the organization to define and agree on specific Sustainable IT objectives that were aligned with the organization's overall sustainability strategy, objectives, and goals. The core of the Intel IT strategy is to increase their sustainable practices and decrease the $\mathrm{CO}_{2}$ footprint of IT and the wider business. As part of alignment with the overall Intel Sustainability Strategy, Intel IT had two critical roles:

- an active contributing role in reducing Intel's environmental impact by aligning IT processes and practices with the core principles of sustainability, which are to reduce, reuse, and recycle; and

- an innovative enabling role by use of IT in business processes to deliver sustainability benefits across the enterprise to manage and improve Intel's environmental performance.

\section{Sustainable IT roadmap}

In order to operationalize their Sustainable IT strategy, the IT Sustainable Program Office defined a long-term ecotechnology roadmap. The goal of the roadmap was to coordinate sustainability initiatives and drive IT's sustainability project and innovation portfolio.

The roadmap includes projects on 'enabling Intel sustainability' covering both reduction projects that reduce the overall footprint, and avoidance projects that keep the footprint from escalating, along with projects on policy and compliance. The decision was taken to have three phases in the roadmap to help Intel IT maintain their focus and balance between sustainability efforts and ongoing business demands. The roadmap phases were:

- Phase 1: Quantify and highlight the (potentially unknown) sustainability impacts of existing IT projects. In most cases, these projects had already been undertaken for costreduction purposes. The resulting analysis provided a baseline of sustainability projects already in progress with measurable environmental reductions.
- Phase 2: Develop innovative proof-of-concepts that can demonstrate new footprint reductions within a 12-month time frame. Identify opportunities outside of IT where IT solutions can reduce Intel's overall footprint in areas such as office buildings, factories, and collaboration technologies.

- Phase 3: Proliferate sustainable IT principles and practices with a focus on using IT to help reduce Intel's carbon footprint across the organization. Phase 3 includes the establishment of a research and development program to explore new concepts and continually optimize the eco-technology roadmap. This phase would include developing a long-term sustainability research strategy.

\section{Understanding the IT footprint}

Intel IT developed a measurement model to provide reliable data to guide both strategic and operational decision-making. Initial calculations estimated the IT equipment footprint to be about $8 \%$ of Intel's overall $\mathrm{CO}_{2}$ emissions. The measurement model enables Intel IT to analyze their direct IT carbon footprint in more detail - considering both where equipment is housed and differing equipment types. The equipment-type analysis, illustrated in Figure 2, enabled Intel IT to categorize equipment into segments that they could evaluate for potential savings with targeted improvement initiatives.

As shown in Figure 3, Intel IT's carbon footprint breakdown was as follows:

- 70\% from data centers. Data centers play a key role in supporting new technology development. Equipment (servers, network, storage, UPS, cooling, etc.) located within the data centers was responsible for the majority of emissions.

- $24 \%$ from computing and information and communication technology (ICT) equipment located outside data centers. Intel is a global company with many sites across the world, and ICT equipment (network, telephony, video conferencing, etc.) plays a critical role in connecting Intel's workforce.

- 6\% from office client computing. Office client (monitors, desktops, laptops, printers, etc.) footprint is quite low, relative to Intel's larger-than-typical data center computing needs, due to the widespread adoption of laptops across the enterprise. More than $80 \%$ of Intel's workforce uses low-energy laptops and monitors.

These three areas provide the highest impact in terms of sustainability benefits and cost considerations. For each focus 


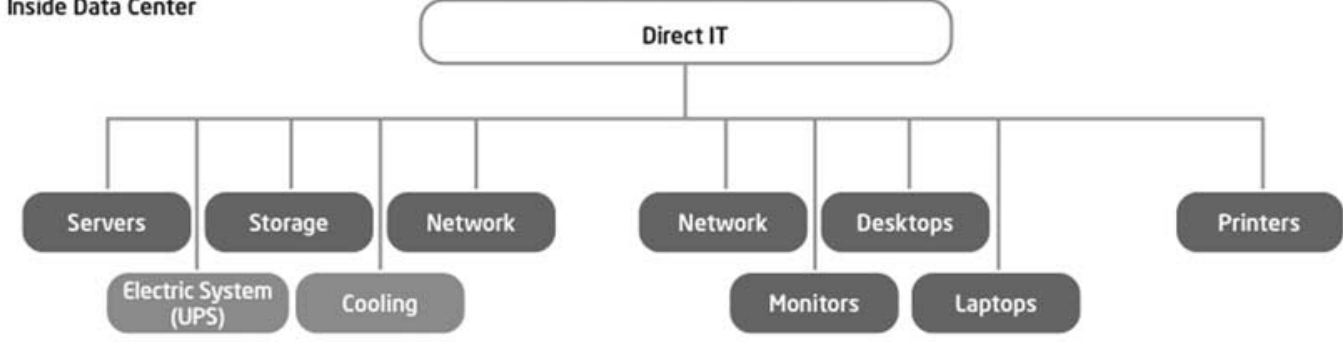

Power Consumption Variables

- Number of Devices

- Power Draw by Model

- Power Management

- Cooling Efficiency

- Uninterruptible Power Supply (UPS) Efficiency
Power Consumption Variables

- Number of Desktops and Laptops

- Power Draw by Model

- User Behavior (Active, Idle, Sleep, Off)

- LCD/CDT Ratio

Figure 2 Overview of devices that contribute to Intel IT's direct carbon footprint.

\section{Direct IT Carbon Footprint Estimate by Equipment Type}

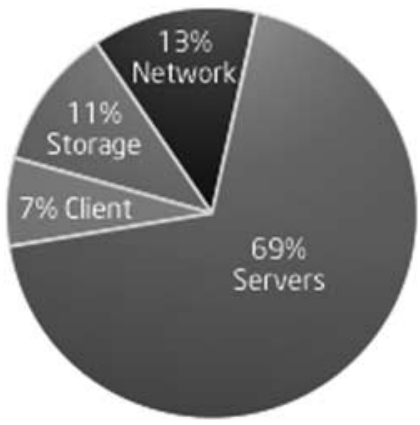

Figure 3 Sources of carbon emissions from Intel IT.

area, Intel IT identified energy-saving projects aligned to the Sustainable IT roadmap and used the 'what-if' analysis capabilities of the model to calculate the carbon footprint reduction for each project on a quarterly basis.

Identifying high-impact opportunities

As part of their Sustainable IT planning, Intel IT identified and evaluated the business areas within their organization and across Intel that would provide the highest impact in terms of sustainability benefits, the required cost investment, potential returns, and the ability of IT to influence the activity.

Environmental impact was determined using the 'what-if analysis capabilities of the measurement model to calculate the potential carbon footprint reduction. For example, the model helped Intel calculate the effect of refreshing 1000 older servers with 300 newer models. In this scenario, the model predicted Intel could double compute capacity as well as reduce $\mathrm{CO}_{2}$ footprint by about 1400 metric tons annually. These calculations were also augmented with rough approximations using publicly available data and average benchmarks scaled to the size of Intel IT.

The influence of IT on the business area was determined by considering metrics for operational control, buying power,

\section{Direct IT Carbon Footprint Estimate by Equipment Location}
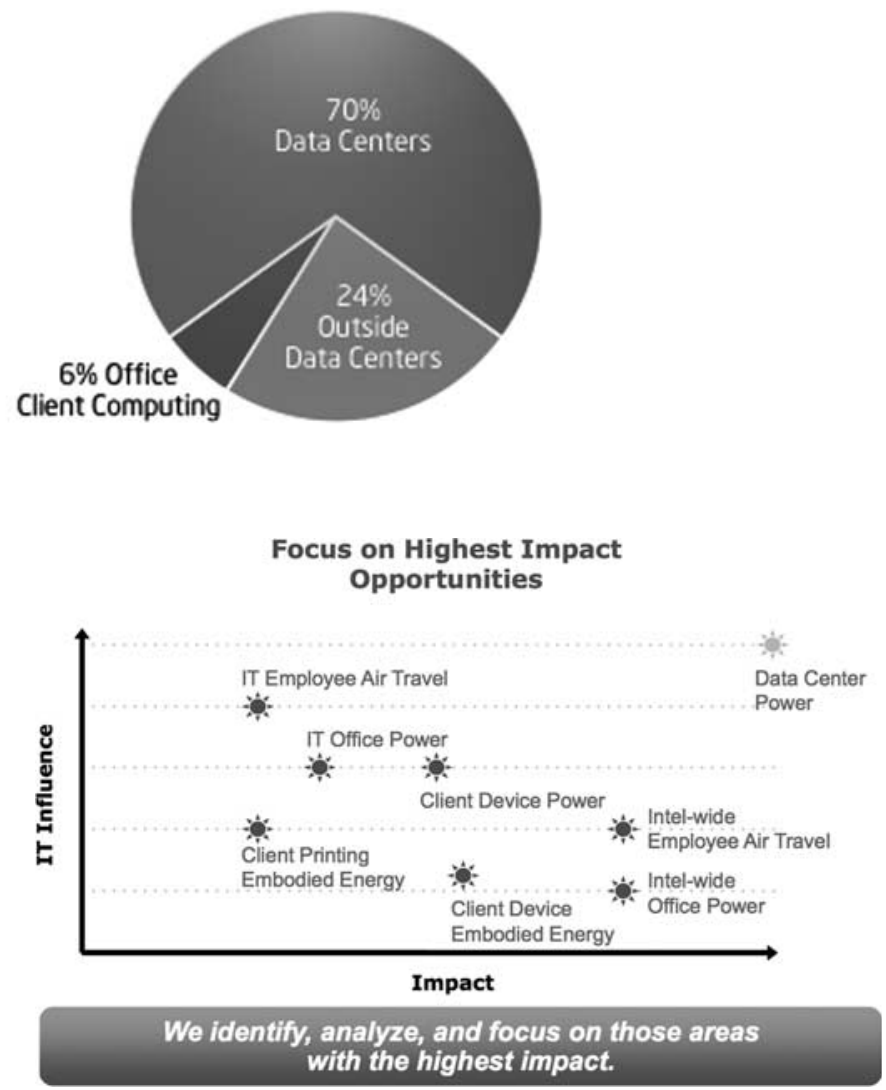

Figure 4 Analysis of sustainability opportunities with highest impacts.

degree of IT enablement, availability of viable alternatives, and other similar factors. The results of this analysis are shown in Figure 4. While it is not feasible to undertake all possible initiatives, the analysis was beneficial within the decision-making process to determine the best places for investment. 
The analysis revealed that inside IT, the largest impact is electricity usage and the carbon emissions associated with its generation. The main potential was on data center power and cooling efficiency. Since the majority of Intel IT carbon emissions are caused by servers, regularly refreshing servers, consolidating applications to common platforms, and turning off unused systems would have the greatest benefits. Outside of IT, the main potential was through the use of communication and collaboration technologies to reduce Intel-wide employee travel.

Develop a sustainable culture

Building a sustainability mindset strengthens business efficiency, reduces consumption and waste, and enhances the Intel brand. Intel knew they would need to influence corporate

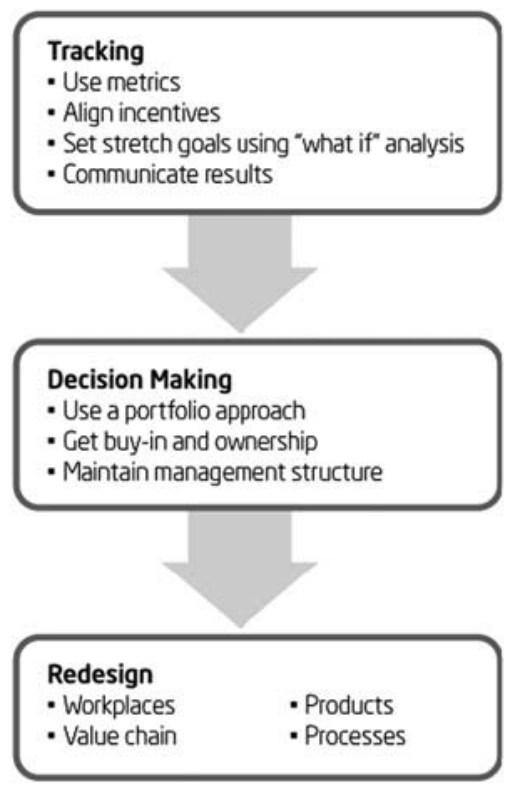

Figure $\mathbf{5}$ Fostering a sustainability culture. culture, including decision-making processes and employee involvement, to be more sustainability-focused and adapt to new thinking and methodologies. The goal was to develop a cohesive, integrated strategy that created awareness along with a sense of urgency within the IT organization and across Intel. The strategy, outlined in Figure 5, employed three key steps focusing on the tracking, decision making, and redesign, to foster a sustainability culture within IT.

IT sustainability principles

To drive adoption, Intel IT developed a clear set of IT Sustainability Principles for their activities. The principles, described in Table 1, are a set of aspirational statements to provide guidance on Sustainable IT practices. The principles play an important role in decision making and are included in measurement models, standards, and processes. The criteria set out by the principles may also influence programs toward suppliers with sustainable business practices.

Encourage creative involvement and innovation from all employees

It is important to drive adoption, create awareness, and practical relevance for all employees, not just specialists in specific projects. In 2009, Intel launched a new environmental employee portal, as well as an interactive online employee community, 'Green Intel,' to facilitate discussion between teams and individuals on sustainability topics such as power management. More than 2000 employees joined Green Intel in the first 6 months, making it the fastest-growing employee group on Planet Blue, Intel's internal social networking platform.

\section{Increase awareness}

Creating awareness is an important part of changing the culture to be more consensus of sustainability. Initially, the IT Sustainability Office had planned an aggressive communication campaign to raise awareness and drive adoption. In order to establish a baseline to measure the results of the campaign, an office awareness study was conducted to

Table 1 Intel IT sustainability principles

Principle Example actions

Consciously Manage Our Capabilities

Include sustainability value and impacts in our proposals, measurements, and

Select Sustainable Suppliers decision making

Work with our supplier managers to ensure our purchases represent and support sustainable business practices

Enable Intel to meet Global Sustainability Proactively monitor global regulations and requirements to ensure IT's and Compliance Intel's compliance

Measure, Monitor, and Optimize Consumption Reduce consumption and actively manage resources using sustainability metrics Enable Sustainable Facilities Work with Technology and Manufacturing Group (TMG) and Corporate Services (CS) to use IT capability to reduce resource consumption within TMG and CS

Enable IT Sustainability Behavior

Enable Travel Avoidance

Promote IT's Sustainability innovations across Intel and externally
Create global awareness of IT Sustainability, which encourages IT employees to be corporate role models

Showcase all IT collaboration technologies across Intel

Support sustainability innovations in our IT solutions, and share those successes across Intel and externally 
determine how strong the corporate culture was in terms of sustainability. The study showed that Intel employees already had a strong sustainability mindset and their direct IT touch was already very efficient. Based on the survey, the IT Sustainability Office focused its efforts in the area of employee incentivization.

\section{Use employee incentive programs to drive innovation}

In 2008, to help focus all of their employees on environmental sustainability, Intel aligned a portion of every employee's variable compensation, from entry-level employees to the $\mathrm{CEO}$, to the achievement of environmental sustainability metrics. Each business unit within Intel had specific goals/ targets to achieve that contribute a specific percentage toward their employee bonus. In 2011, the environmental metrics focused on energy efficiency in operations and for new products; IT Energy reduction targets were the component of IT employee bonus schemes.

The alignment of the employee bonus has proved to be the prime incentive to engage and facilitate employee involvement to help them collectively focus on the importance of achieving the organization's environmental objectives. The employee bonus encourages employees to examine projects they are currently working on to identify tangible contributions toward sustainability goals.

Build the business case for sustainability

Intel IT has discovered there is a strong business case for sustainable computing, with cost savings from Sustainable IT projects (i.e. energy savings from data centers, travel cost avoidance for video conferencing) justifying investment.
It is important to build a strong business case for Sustainable IT projects. Return on Investment is king and business incentives are critical to getting the support needed for projects. This is the main difference between a Green IT program (environmental benefits) and a Sustainable IT program (environmental and business benefits). From the experience within Intel, making the business case for sustainability within green fields projects is straightforward - it makes sense to build a new Data Center to Best Known Methods. It is much more challenging to determine the business case within a retrofit scenario, here sustainability can become hard and expensive and it needs to have a solid sustainable business case.

\section{Impacts of sustainable IT @ Intel}

Since the CEO set the 2007 objectives, the Intel sustainability program has had encouraging results as detailed in Table 2. At the end of 2011, Intel had reduced their absolute emissions more than $60 \%$ below 2007 levels. In the same time frame, Intel annual revenue increased by $41 \%$.

The IT sustainability program has allowed Intel IT to increase the performance of its computing environment while reducing the overall IT carbon footprint. This has resulted in energy cost savings of $\$ 5.8$ million in 2010 (up from $\$ 4$ million in 2009) and the avoidance of more than 60,000 metric tons of $\mathrm{CO}_{2}$ emissions. The overall performance of Intel IT is detailed in Table 3.

\section{Green for IT: improving IT energy efficiency}

In the last 3 years, Intel IT has increased the performance of their computing environment in areas of compute capacity,

Table 2 Intel overall corporate sustainability performance, 2007-2011

\begin{tabular}{lccccc}
\hline Intel-wide performance & 2007 & 2008 & 2009 & 2010 & 2011 \\
\hline Net Revenue (US dollars in billions) & $\$ 38.3$ & $\$ 37.6$ & $\$ 35.1$ & $\$ 43.6$ & $\$ 54.0$ \\
Workplace employees at year end & 86,300 & 83,900 & 79,800 & 82,500 & $100,100^{\mathrm{a}}$ \\
Greenhouse gas emissions (million metric tons of $\mathrm{CO}_{2}$ equivalent) & 3.85 & 2.75 & 2.05 & 2.12 & 1.40 \\
Energy use (billion kWh - includes electricity, gas, and diesel) & 5.8 & 5.6 & 5.1 & 5.2 & 5.3 \\
Revenue per metric ton of $\mathrm{CO}_{2}$ equivalent (dollars) & $\$ 9948$ & $\$ 13,673$ & $\$ 17,122$ & $\$ 20,566$ & $\$ 38,571$ \\
Energy use per employee (kWh - includes electricity, gas, and diesel) & 66,709 & 67,330 & 64,073 & 62,933 & 52,947 \\
\hline
\end{tabular}

${ }^{\text {a }}$ Total employee count includes wholly owned subsidiaries that Intel IT does not directly support. Intel IT directly supported 91,500 employees in 2011.

b Including renewable energy credit purchases.

Source: Intel APRs '07-'11.

Table 3 Intel IT performance, 2009-2011

\begin{tabular}{|c|c|c|c|}
\hline Intel IT & 2009 & 2010 & 2011 \\
\hline IT employees & 5660 & 6300 & 6400 \\
\hline IT spend (as \% or net revenue) & $3.6 \%$ & $3.0 \%$ & $2.6 \%$ \\
\hline IT spending per employee & $\$ 16,100$ & $\$ 16,400$ & $\$ 15,500$ \\
\hline IT $\mathrm{CO}_{2}$ footprint & 253,000 & 249,000 & 246,000 \\
\hline IT spending (dollars in millions) & $\$ 1264$ & $\$ 1308$ & $\$ 1404$ \\
\hline Saving from travel avoidance due to videoconferencing & $\$ 14$ & $\$ 27$ & $\$ 73$ \\
\hline $\mathrm{CO}_{2}$ saving from travel avoidance due to videoconferencing (metric tons of $\mathrm{CO}_{2}$ equivalent) & N/A & 22,500 & 65,000 \\
\hline
\end{tabular}

Source: Intel APRs, Intel IT APRs '09-'11. 
Table 4 Intel operational performance, 2008-2011

\begin{tabular}{lcc}
\hline Intel IT & 2009 & 2010 \\
\hline IT data centers & 95 & 91 \\
Storage capacity (petabytes) & 18.6 & 24.9 \\
Internet network bandwidth (gigabits per sec) & 3.0 & 4.8 \\
Compute capacity for silicon design & $24 \%$ & $84 \%$ \\
(growth from 2008) & 14 days & 38.2 \\
Time to deploy infrastructure services & $12 \%$ & 4.2 \\
Percentage of applications virtualized & & $42 \%$ \\
(in Office and Enterprise environments) & $64 \%$ \\
\hline
\end{tabular}

Source: Intel APRs, Intel IT APRs '08-'11.

storage, and network as detailed in Table 4, and are providing IT services to an additional 11,000 employees, all while reducing the overall carbon emissions of IT operations.

One of the primary ways Intel IT is delivering reductions in its carbon footprint is through server consolidation. In keeping up with Moore's law and ever increasing computing demand, Intel IT have settled on a 4 -year server refresh policy. One server with the latest processing technology can replace as many as 10 four year-old servers. The refresh strategy has allowed them to double compute capacity and lower costs. In 2010, server refresh saved an estimated 28,000 metric tons in $\mathrm{CO}_{2}$; this is the equivalent of the carbon captured by 8000 acres of pine forest in a year.

Intel IT have also introduced an aggressive server virtualization strategy that has increased the utilization of their servers, leading to a further 2500 metric tons of carbon footprint reduction in 2010. Improving the management of workload distribution of servers within the data center has allowed Intel IT to optimize server utilization, further allowing them to power off over 2300 servers. Overall, efficiency efforts at Intel IT have reduced the number of servers from a high of $100 \mathrm{k}$ to $75 \mathrm{k}$.

Within the area of client computing, they have achieved a $70 \%$ saving per user by deploying low-power laptops over desktops, and have over $80 \%$ laptop deployment within their environment. They are deploying Solid State Drives (SSDs) that consume less power than conventional hard drives in their laptops. SSDs are also being considered for use in the data center, where they will save energy and reduce heat while enhancing performance. Overall, Intel IT has saved over 100 million kilowatt-hours (kWh) in energy.

IT for green: reducing travel with collaboration technologies In 2011, Intel had over 91,500 employees across 164 sites in 62 countries around the world. These employees typically work in distributed and virtual teams. The cost of employee travel is expensive in both financial and environment terms. Intel IT makes extensive use of collaboration technology, such as video conferencing, which can reduce the need to travel.

For person-to-person and small group meetings desktopbased videoconferencing is provided. Desktops are refreshed at Intel to ensure they have the capacity to handle full-screen video and application sharing. Meetings of larger teams and groups are accommodated with the use of telepresence rooms. During 2010, Intel more than tripled the number of meeting rooms with videoconferencing capabilities, including the addition of rooms in 11 new countries. The estimate for 2011 was that videoconferencing and telepresence rooms would save 435,000 employee travel hours, avoiding more than $\$ 73$ million in travel expenses, and over 65,000 metric tons of $\mathrm{CO}_{2}$ emissions.

\section{Towards 2020}

As Bill Guyon assesses the existing strategy for IT Sustainability, he can point to many achievements. Using Sustainable IT, Intel IT has optimized the use of data center, compute, and office infrastructure to reduce their energy consumption, while leveraging collaboration technologies to reduce the need for employees to travel. Overall, these efforts have resulted in cost savings of over $\$ 114$ million and the avoidance of more than 87,500 metric tons of $\mathrm{CO}_{2}$ emissions. In recognition of Intel IT's sustainability achievements, Intel was named to Computerworld's 2010 and 2011 lists of 'Top Green-IT Organizations.' However, significant challenges exist.

Intel IT's initial strategy has utilized employee benefit scheme as a compensation motivator to embed the sustainability within the cultural mindset. However, as employees exploit their current projects for sustainability gains, the 'low-hanging fruit' will eventually be exhausted. Intel is seeing diminishing returns as they optimize their operations. For example, take data centers; the introduction of a private cloud will drive down server counts significantly, along with associated energy usage, to a critical mass running at high utilization, after that further energy reductions will be based on refresh cycles.

Guyon needs to decide what are the next strategic steps for Sustainable IT at Intel. With the short-term low-hanging fruit all picked, a new strategic focus is now needed. He needs to answer a key question. Should Sustainable IT continue on its existing path (business-as-usual), or should Intel look to further exploit the transformational and innovation potential of next-generation Sustainable IT technologies within the organization and across the wider extended-enterprise? Should Guyon propose a strategic shift to investing in the next generation of sustainable ICT technologies and in particular opportunities outside of IT, where IT solutions could help reduce Intel's carbon footprint across the organization? This requires the development of a long-term sustainability research strategy together with the establishment of a research and development program to explore new concepts and continually optimize the eco-technology roadmap. The challenge with such a program is to get management commitment, as they will question the need to allocate incremental resources to 
drive harder on medium- and long-term projects when they can still see short-term projects still delivering results.

Is Intel IT on track with its Sustainable IT plan? Will their current three-phase roadmap endure? Is Intel at par, ahead, or behind the competition in terms of sustainability? How can Sustainable IT continue to add value at Intel? What are the opportunities the next generation of sustainable IT technologies present? Can Intel work with its supply chain partners to reduce global carbon emissions? How can Intel become more competitive through the next generation of sustainable production? What should be Intel's strategy going forward? What are the strengths, weaknesses, opportunities, and threats for IT Sustainability at Intel? Can Sustainable IT be a competitive advantage for Intel?

\section{Acknowledgements}

The work presented has been funded by Science Foundation Ireland under Grant No. SFI/08/CE/I1380 (Lion-2) and by Enterprise Ireland under Grant CC/2009/0801.

\section{Note}

1 Creative license has been used within the scenario.

\section{References}

Climate Group. (2008). SMART 2020: Enabling the low carbon economy in the information age, The Climate Group London, http://www.smart2020.org/_assets/ files/02_Smart2020Report.pdf.

Curry, E., Guyon, B., Sheridan, C. and Donnellan, B. (2012). Developing an Sustainable IT Capability: Lessons from Intel's journey, MIS Quarterly Executive 11(2): 61-74.

Curry, E., Guyon, B., Sheridan, C. and Donnellan, B. (2012). Sustainable IT: Challenges, postures, and outcomes, IEEE Computer 45(11): 79-81.

Donnellan, B., Sheridan, C. and Curry, E. (2011). A Capability Maturity Framework for Sustainable Information and Communication Technology, IEEE IT Professional 13(1): 33-40.

Elliot, S. (2007). Environmentally Sustainable ICT: A critical topic for IS research? in Pacific Asia Conference on Information Systems (PACIS 2007); Auckland, New Zealand: Association for Information Systems, 100-114.
Enkvist, P.-A. and Rosander, J. (2007). A Cost Curve for Greenhouse Gas Reduction, The McKinsey Quarterly 34.

Murugesan, S. and Laplante, P.A. (2011). IT for a Greener Planet, IEEE IT Professional 13(1): 16-18.

Porter, M.E. and Linde, C.V.D. (1995). Toward a New Conception of the Environment-Competitiveness Relationship, Journal of Economic Perspectives 9(4): 97-118.

\section{About the Authors}

Edward Curry is a research scientist and leads the Green and Sustainable IT research group at the Digital Enterprise Research Institute (http://www.deri.ie). His research projects include studies of sustainable IT, energy intelligence, semantic information management, and collaborative data management. Curry has worked extensively with industry and government, advising on the adoption patterns, practicalities, and benefits of new technologies. He has published in leading journals and books, and has spoken at international conferences including the MIT CIO Symposium. He currently participates in a project for the European Commission to define a research strategy for the Big Data economy within Europe. He has a Ph.D. from the National University of Ireland, Galway (http://www.nuigalway.ie) and serves as an adjunct lecturer within the university.

Brian Donnellan is Professor of Information Systems Innovation at the National University of Ireland, Maynooth (http:// www.nuim.ie) and Academic Director of the Innovation Value Institute (http://www.ivi.ie). Prior to joining National University of Ireland, Maynooth, Professor Donnellan was a faculty member in the National University of Ireland, Galway. Earlier, he spent 20 years working in the ICT industry, where he was responsible for the provision of IS to support product development. He is an expert evaluator for the European Commission and has been guest and associate editor of several leading IS journals including the Journal of IT, Journal of Strategic Information Systems, and MIS Quarterly. 